GUÍA DE PRÁCTICA<smiles>c1ccc2ccccc2c1</smiles>

\title{
GUÍA PRÁCTICA PARA LA TOMA DE IMPRESIÓN EN ALGINATO
}

Apropiación social del conocimiento

Generación de contenidos impresos http://repository.ucc.edu.co/handle/20.500.12494/7375 N. ${ }^{0} 13$, diciembre de 2019 doi: https://doi.org/10.16925/gcgp.18

Claudia Elena Restrepo-Villamizar

Universidad Cooperativa de Colombia

Sede Villavicencio

\section{NOTA LEGAL}

El presente documento de trabajo ha sido incluido dentro de nuestro repositorio institucional como Apropiación social de conocimiento por solicitud del autor, con fines informativos, educativos o académicos. Asimismo, los argumentos, datos y análisis incluidos en el texto son responsabilidad absoluta del autor y no representan la opinión del Fondo Editorial o de la Universidad.

\section{DISCLAIMER}

This coursework paper has been uploaded to our institutional repository as Social Appropriation of Knowledge due to the request of the author. This document should be used for informational, educational or academic purposes only. Arguments, data and analysis included in this document represent authors' opinion not the Press or the University. 


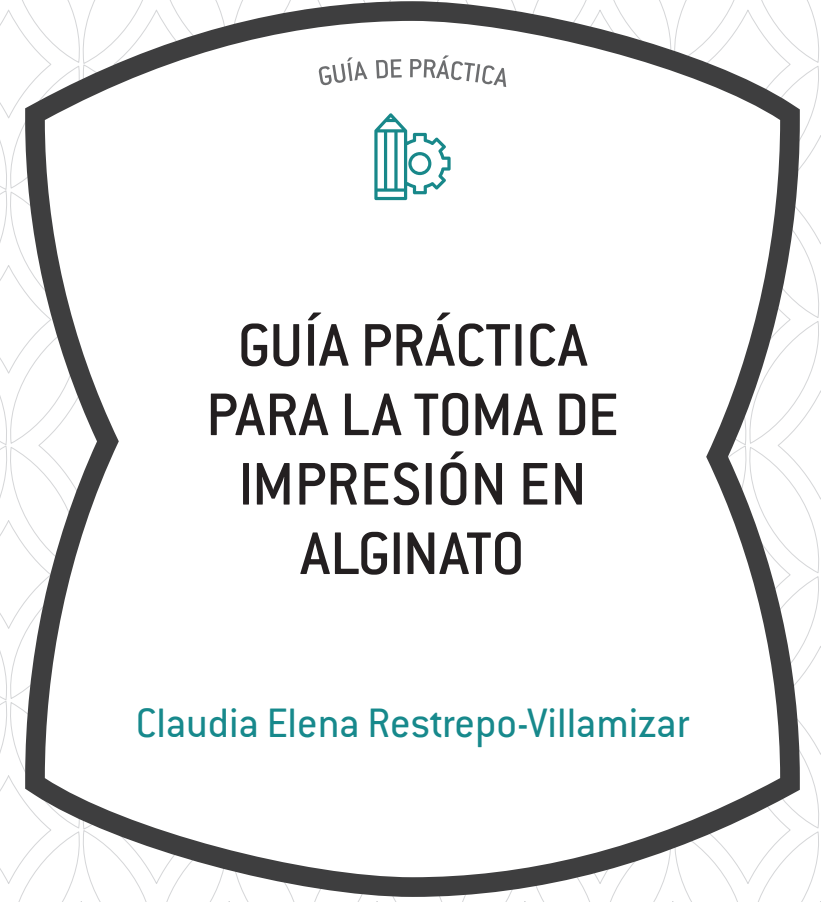




\section{ACERCA DE LA AUTORA}

Claudia Elena Restrepo-Villamizar, odontóloga especialista en Salud Familiar, en proceso de maestría en Educación. Profesora instructora del programa de Odontología, Universidad Cooperativa de Colombia, sede Villavicencio, Colombia.

Correo electrónico: claudiae.restrepo@campusucc. edu.co

ORCID: https://orcid.org/0000-0002-8308-5913

\section{CÓMO CITAR ESTE DOCUMENTO}

Restrepo-Villamizar, C. E. (2019). Guía práctica para la toma de impresión en alginato (Generación de contenido impreso N.¹3). Bogotá: Ediciones Universidad Cooperativa de Colombia. doi: https://doi. org/10.16925/gcgp.18.

Este documento puede ser consultado, descargado o reproducido desde nuestro repositorio institucional (http://repository.ucc. edu.co/handle/20.500.12494/7369) para uso de sus contenidos, bajo la licencia de Creative Commons Reconocimiento-No Comercial-Sin Obra Derivada 4.0 Internacional. http://creativecommons.org/licenses/by-nc-nd/4.0/

\section{(c) $(9 \Theta$}




\section{TABLA DE CONTENIDO}

INTRODUCCIÓN

$\begin{array}{lr}\text { RECOMENDACIONES } & 6\end{array}$

$\begin{array}{ll}\text { Oвjetivos } & 6\end{array}$

Objetivos Generales $\quad 6$

$\begin{array}{ll}\text { OBJETIVOS ESPECÍfICOS } & 6\end{array}$

$\begin{array}{ll}\text { MATERIALES E INSTRUMENTAL } & 7\end{array}$

$\begin{array}{ll}\text { REgLAMENTO } & 7\end{array}$

DESCRIPCIÓN DE LA ACTIVIDAD Y PROCEDIMIENTO PRÁCTICO 7

$\begin{array}{ll}\text { CONCLUSIONES } & \text { IO }\end{array}$ 


\title{
13 GUÍA PRÁCTICA PARA LA TOMA DE IMPRESIÓN EN ALGINATO
}

\author{
Claudia Elena Restrepo-Villamizar
}

\section{Resumen}

El objetivo de la guía de toma de impresión en alginato es ayudar al estudiante a conocer el proceso de la toma de una impresión, a fin de que pueda reproducir una réplica fiel de los tejidos duros y blandos de la cavidad oral, con el uso del material hidrocoloide denominado "alginato". Lo anterior teniendo en cuenta los más mínimos detalles a la hora de la toma, de tal forma que se puedan registrar todos los componentes y las características de las piezas dentales.

Palabras clave: alginato, cavidad oral, cubeta, impresión. 


\section{INTRODUCCIÓN}

Una impresión es una réplica fiel de los dientes y los tejidos de la cavidad oral, y es el resultado del uso de materiales como alginato. Los materiales de impresión son usados para tomar la huella de las estructuras orales, y a su vez suelen tener un estado plástico o fluido durante el proceso de la toma de la réplica. Estos materiales, a pesar de sufrir cambios (como polimerización, fraguado, cambios físicos, reacciones de diversa índole), se terminan convirtiendo en materiales elásticos o rígidos, proporcionándonos una reproducción en negativo de los tejidos duros y blandos de la boca.

\section{RECOMENDACIONES PARA UNA BUENA IMPRESIÓN}

Es bueno dejar claro que el alginato por sí solo no es el factor único para el éxito final de una buena impresión, sino que para alcanzar este éxito también se debe tener en cuenta:

- Hacer adecuada manipulación.

- Conocer las características y exigencias del material para impresión más adecuado.

- Cumplir los requisitos para su utilización, por ejemplo, no dejar destapada la bolsa de alginato.

- Elegir y alistar la cubeta previamente estéril.

- Utilizar solamente productos certificados.

- Almacenar en sitios que no cambien de temperatura.

- No exponerlo a elevadas temperaturas.

Los hidrocoloides irreversibles son de los materiales más manipulados en el área de la odontología. Su uso se fundamenta en la facilidad del manejo del material y la reproducción confiable de estructuras en los distintos campos, en especial de la odontología.

Mostraremos el paso a paso del uso del alginato y la posterior toma de impresión, de modo que el estudiante logre efectuar una adecuada toma de impresión. Esta guía va acompañada de fotos que irán ilustrando la teoría, a fin de que el estudiante pueda alcanzar la competencia de "comparar los materiales dentales (alginato, yesos, ceras) desde los principios biológicos, las propiedades mecánicas y físicas, y su composición".

Durante mucho tiempo, se han implementado distintas maneras de lograr la impresión ideal y con el uso de este material, se obtuvo una manera fácil, rápida y no costosa de copiar fielmente los tejidos dentarios.

\section{OBJETIVOS}

\section{OBJETIVOS GENERALES}

- Conocer la técnica de impresión en alginato para proporcionar mayor conocimiento a los estudiantes.

- Preparar a los estudiantes en lo relacionado con los materiales dentales de uso muy frecuente para que dispongan de razonamientos adecuados para su adecuada elección y uso, con base en la literatura científica.

\section{OBJETIVOS ESPECÍFICOS}

- Identificar la técnica de toma de impresión para conseguir su destreza.

- Lograr la capacidad de decidir sobre el correcto y adecuado uso de los materiales dentales de uso clínico. 


\section{MATERIALES E INSTRUMENTAL}

- Tasa de caucho flexible

- Espátula para hidrocoloides

- Cuchara y frasco probeta para dispensar el material

- Cubetas metálicas perforadas

- Cuchillo para prótesis

- Papel vinipel

- Babero

- Tapabocas

\section{REGLAMENTO}

- Identificar el producto y verificar la originalidad de sus etiquetas.

- Registro Invima.

- Distribuidor confiable, entregas en fechas establecidas, que ofrezcan garantía de sus productos, versatilidad de marcas.

- Fecha de fabricación y expiración del material.

- Seguir las recomendaciones del fabricante.

\section{DESCRIPCION DE LA ACTIVIDAD Y PROCEDIMIENTO DE LA PRÁCTICA}

Paso 1: Tenga listos los materiales.

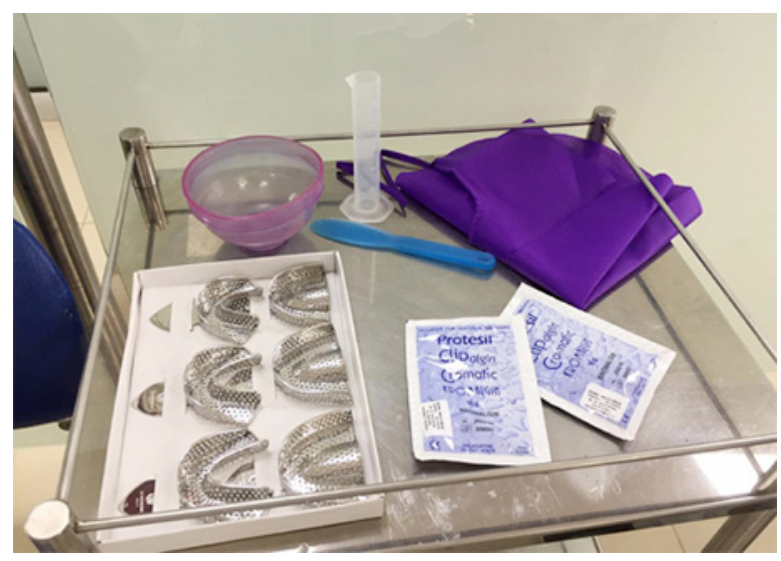

FIGURA 1. Materiales. Fuente: elaboración propia.
Paso 2: Selección de la cubeta.

- Ensaye la cubeta en el maxilar del paciente y seleccione la de tamaño adecuado.

- Preferiblemente, una cubeta con 3 a 4 mm de espacio entre la cubeta y los dientes para lograr con el material mejor volumen, precisión y firmeza.

- La cubeta deberá alcanzar la zona vestibular, hasta copiar del mismo modo los tejidos y cubrir el espacio distal del tercer molar; en cuanto a la cubeta inferior, lo ideal es que alcance la zona distal también del tercer molar.

- Las cubetas metálicas perforadas son las ideales.

- La cubeta debe ser rígida. No es recomendada la cubeta plástica pues alcanza a deformarse al momento de sacarla de la boca, provocando deformación de la impresión.
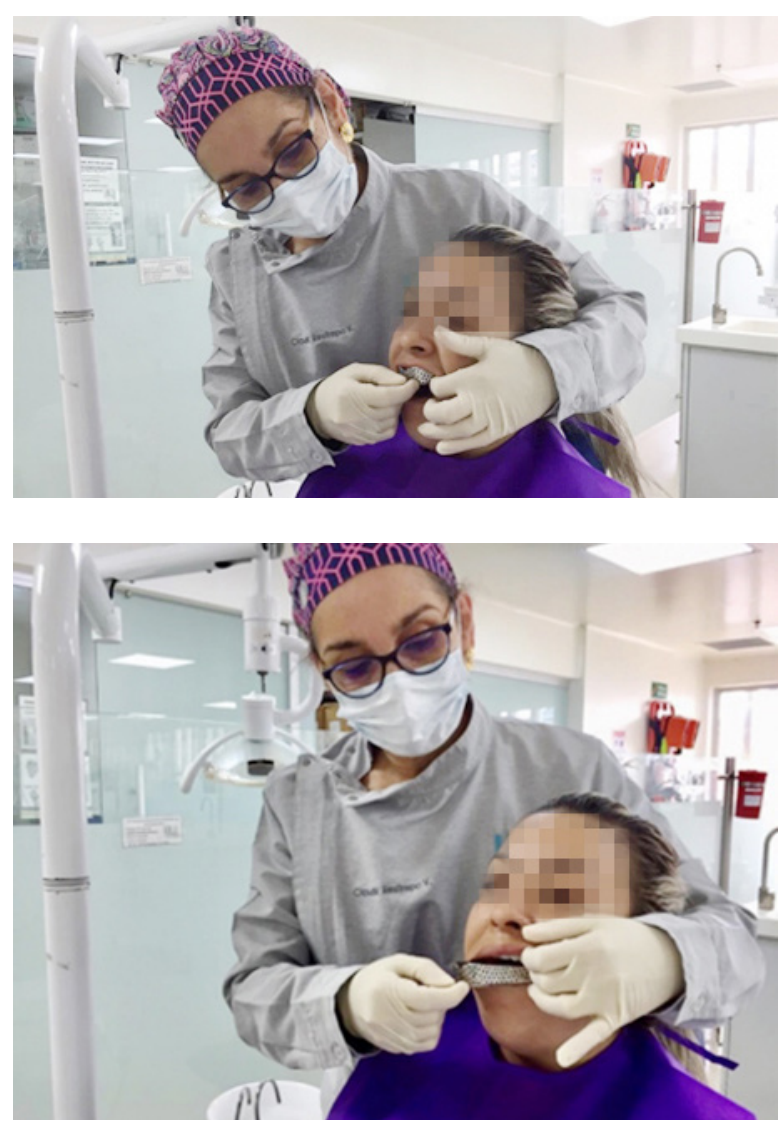

FIGURA 2. Prueba de la cubeta. Fuente: elaboración propia. 
Paso 3. Preparación del paciente.

- Llevamos la cubeta superior a prueba, verifique que no le moleste o tenga sensación de ahogo.

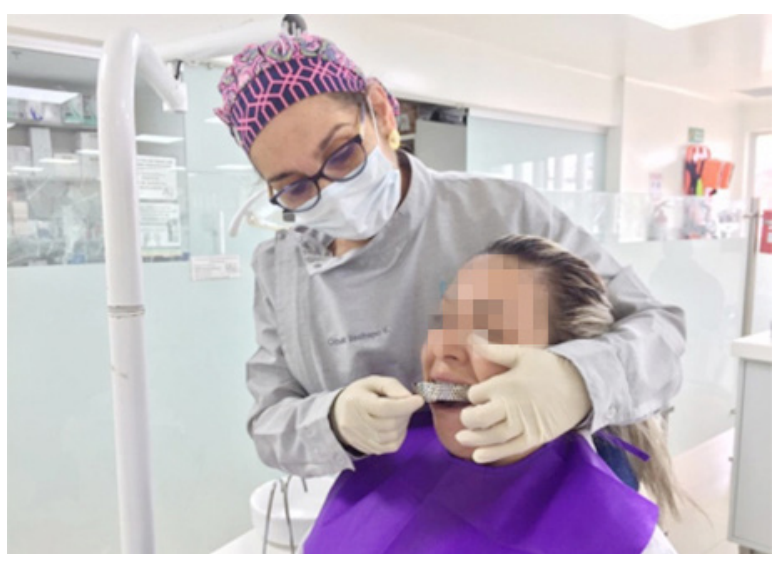

FIGURA 3. Verificación de no molestia. Fuente: elaboración propia.

Paso 4: Toma de impresión

- Agregue de una o dos partes de polvo con el medidor que trae la bolsa.

- Espatule de manera muy vigorosa en dirección a las paredes de la taza de caucho, durante máximo 60 segundos.

- Adicione el alginato dentro de la cubeta.

- Lleve la cubeta a la cavidad oral asegurándose que la orientación de la cubeta coincida con el centro del labio superior del paciente.

- Realice presión hasta que fluya el alginato. Indique al paciente que no respire por la boca.

- Espere aproximadamente dos minutos hasta que el alginato se torne opaco, verifique su gelificación con sus uñas.

- Retire la cubeta de un solo movimiento.

- Proceda a la desinfección de la impresión con espray de glutaraldehido al 2\% o con espray de amonios cuaternarios; o sumergirlo en clorexidina por 60 segundos, nunca hipoclorito porque se degrada.
- Realizar el vaciado.

Dado el caso que no pueda realizar el vaciado en el momento, la impresión debe ser guardada en una bolsa ziploc o envuelta en papel cristaflex por máximo seis horas en clima frío y máximo cuatro horas en clima caliente.

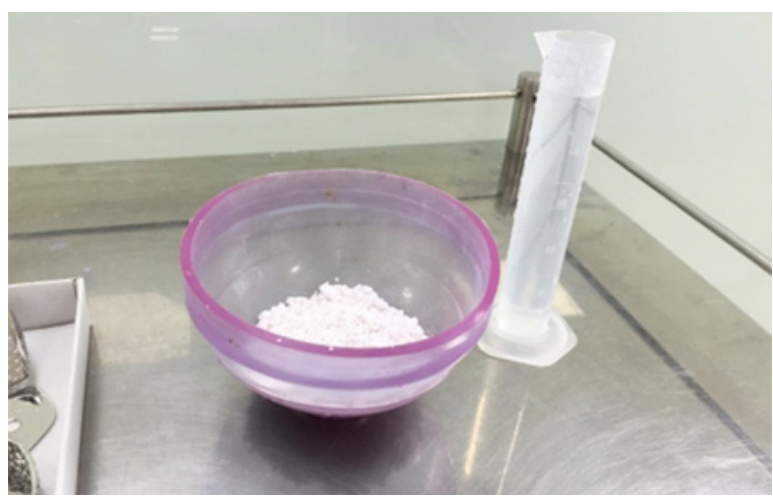

FIGURA 4. Preparación de los insumos. Fuente: elaboración propia.

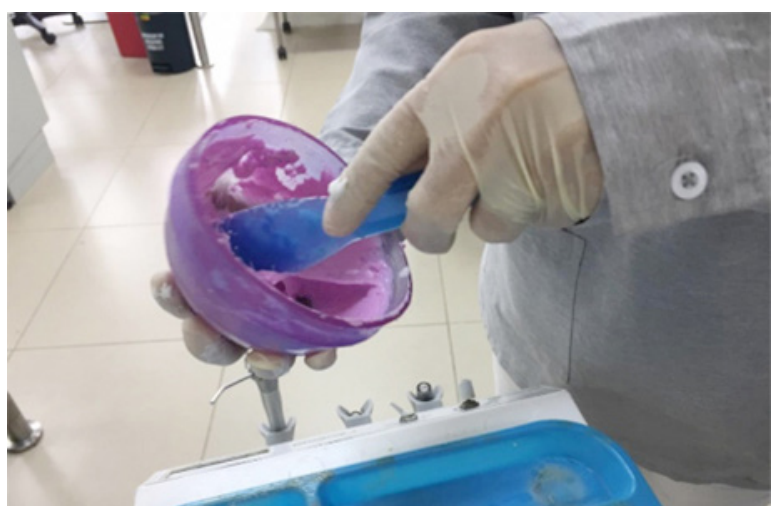

FIGURA 5. Mezcla del agua con el alginato. Fuente: elaboración propia.

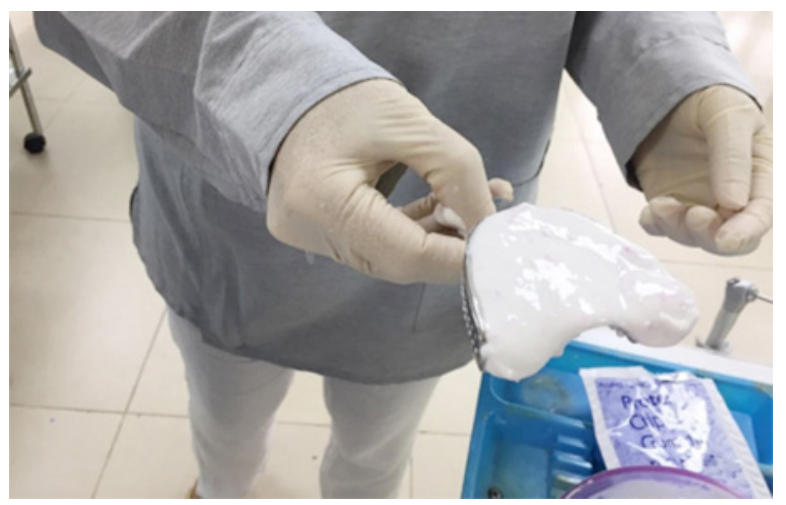

FIGURA 6. Se incorpora el alginato a la cubeta. Fuente: elaboración propia. 


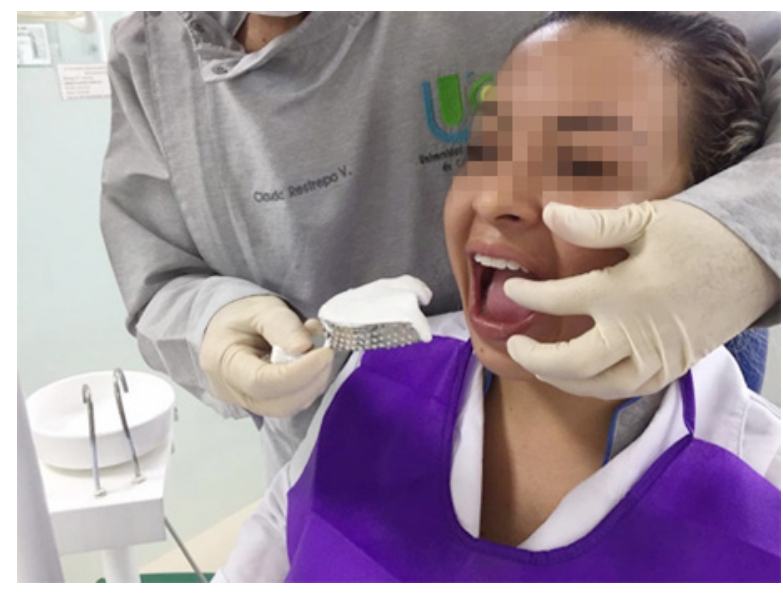

FIGURA ?. Llevamos la impresión a la boca. Fuente: elaboración propia.

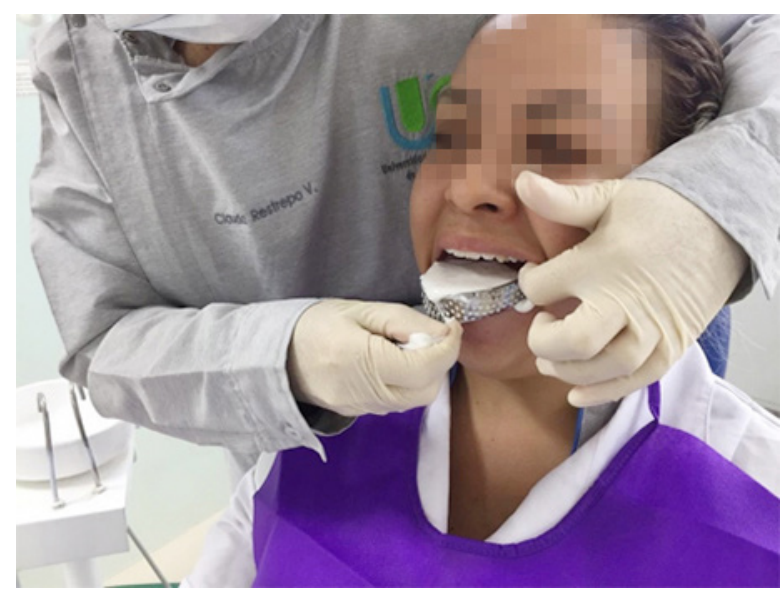

FIGURA 8. Toma impresión superior, sostenemos la impresión por detrás del paciente. Fuente: elaboración propia.

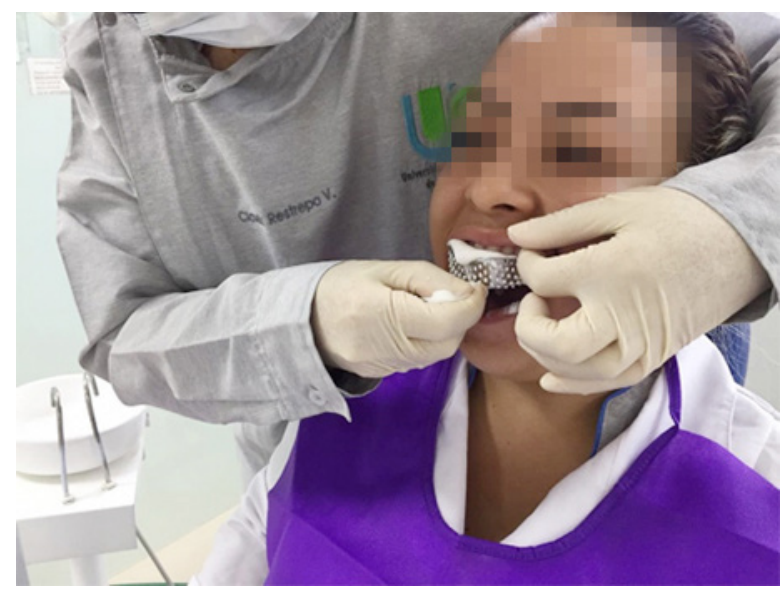

FIGURA 9. Impresión superior. Fuente: elaboración propia.

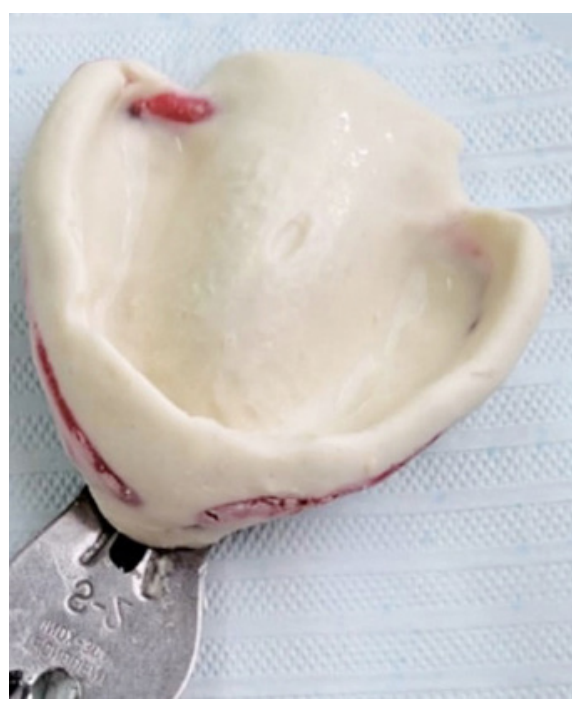

FIGURA 10. Resultado final impresión superior. Fuente: elaboración propia.

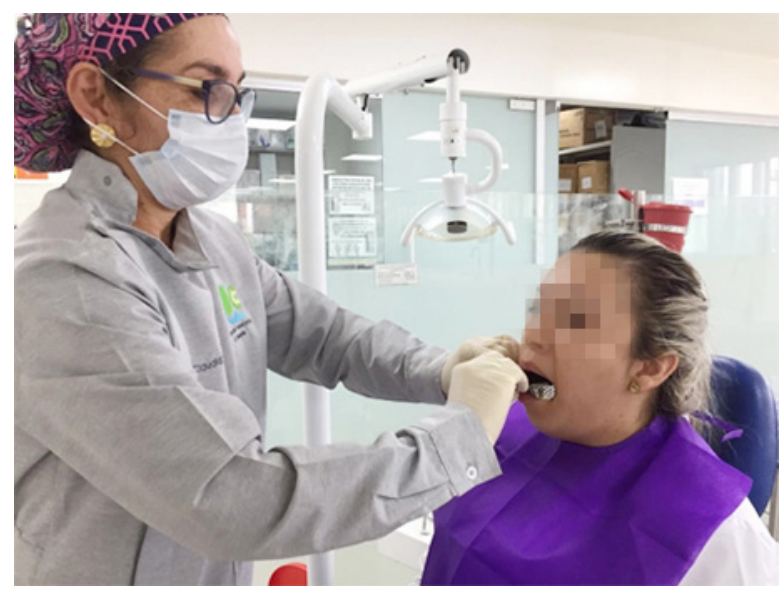

FIGURA 11. Medimos la cubeta inferior Fuente: elaboración propia.

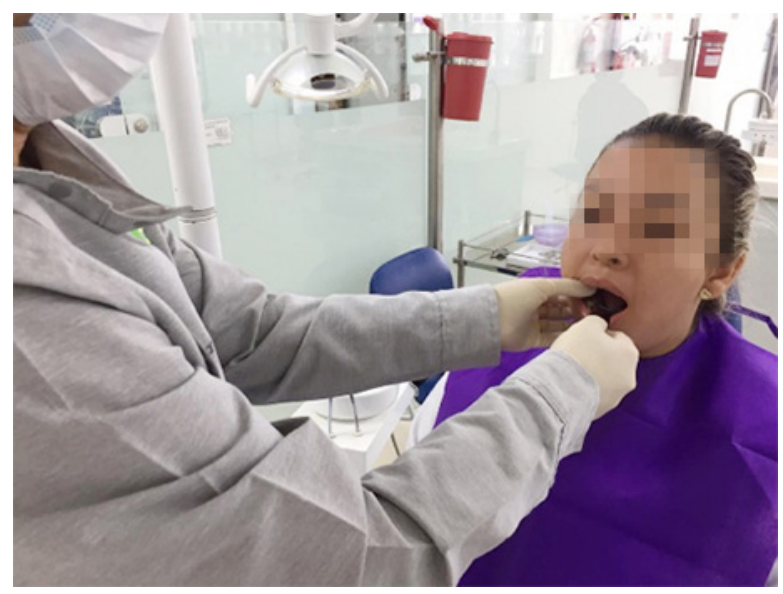

FIGURA 12. Verificamos que no ajuste. Fuente: elaboración propia. 


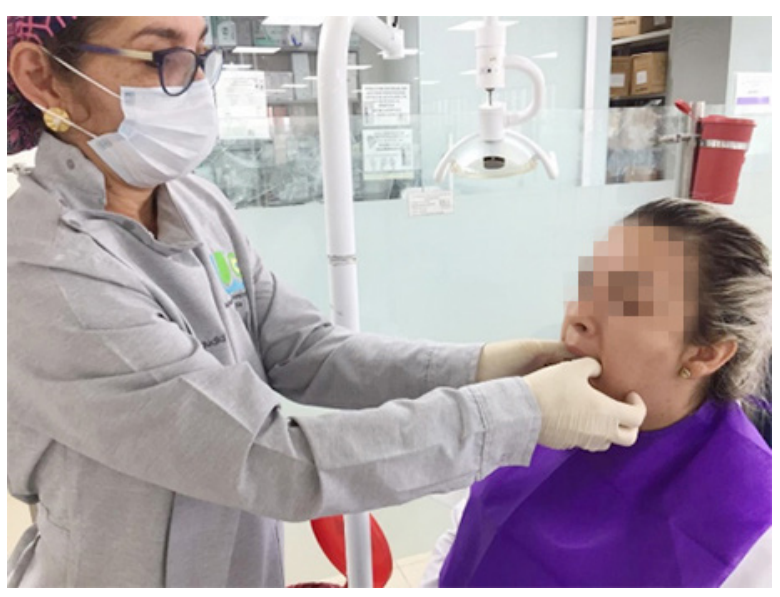

FIGURA 13. Toma impresión inferior, sostenemos la impresión por delante del paciente. Fuente: elaboración propia.

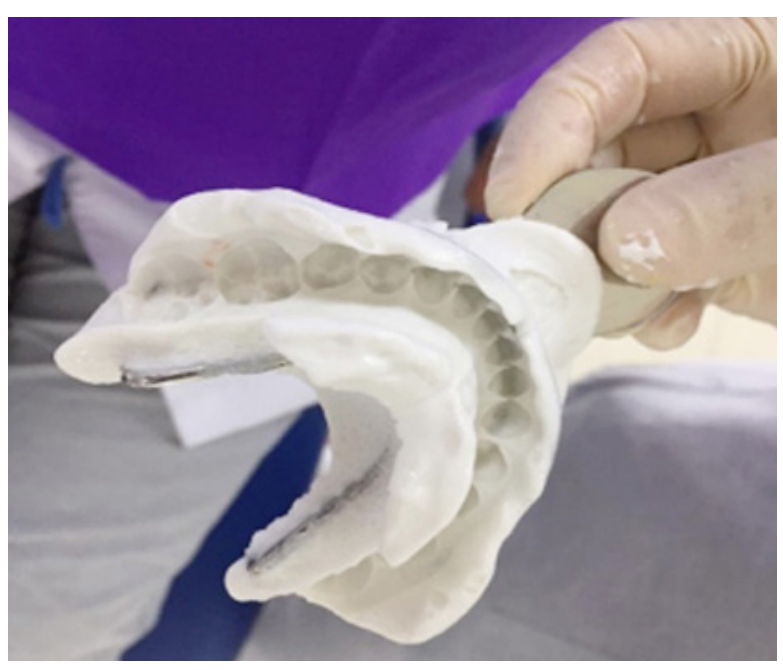

FIGURA 14. Resultado final impresión inferior. Fuente: elaboración propia.
Paso 5: Almacenaje.

- Lo más recomendable es la compra del alginato en bolsas selladas, pues reduce al mínimo la contaminación

- Una vez abierta la bolsa, debe ser guardado de manera hermética.

- Debe agitarse la bolsa del alginato para que la mezcla sea homogénea.

\section{CONCLUSIONES}

Una vez revisada esta guía, el estudiante comprenderá mejor la demostración que el profesor realice y así cada uno podrá ejercer los conocimientos básicos y el dominio del alginato al momento de la toma de impresión.

Así, se alcanza la competencia requerida de "comparar los materiales dentales (alginatos, yesos y ceras), desde los principios biológicos, las propiedades mecánicas y físicas, y su composición”, y su indicador "manipulación del alginato, toma de impresiones y obtención de modelos de trabajo y de estudio". 


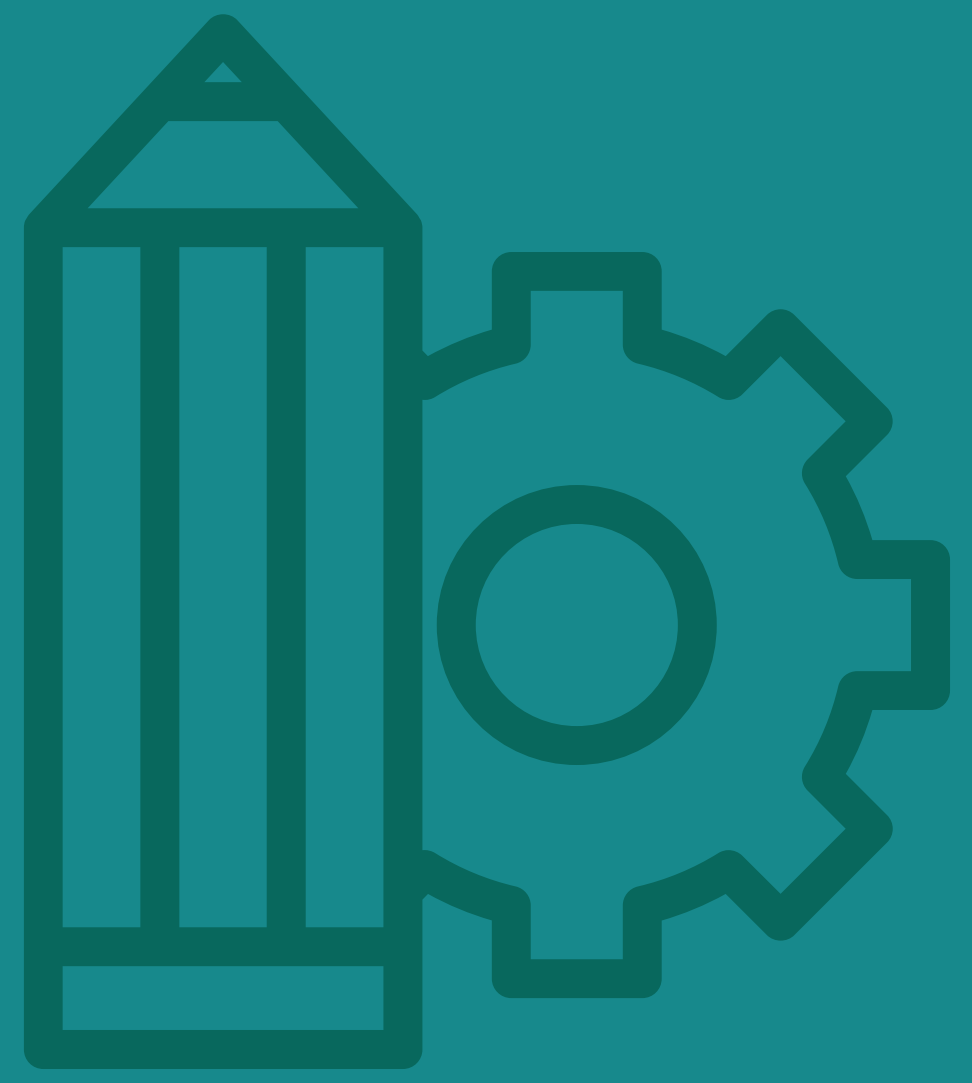

